\title{
A microscopic analysis of single-use versus multi-use phacoemulsification tubing in maintaining sterility and patient safety
}

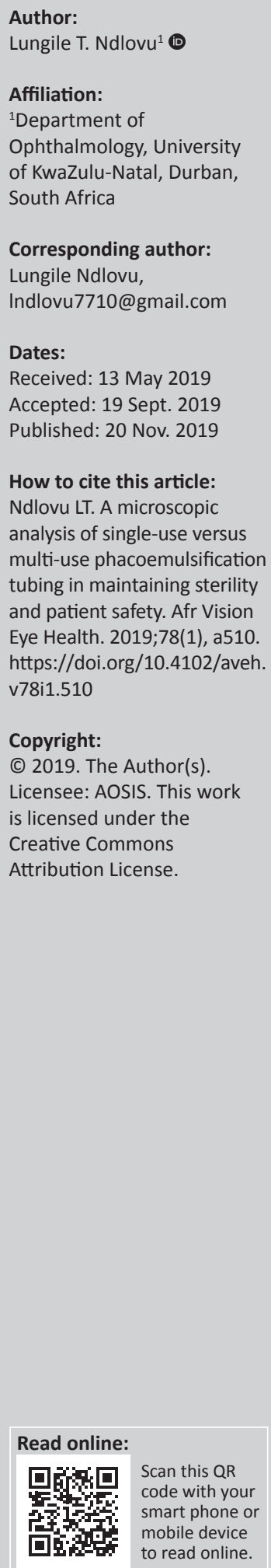

Background: The Pietermaritzburg (PMB) complex in South Africa does not have a uniform protocol regarding reuse of phacoemulsification tubing. Each hospital in the complex has its own guideline, based on manufacturer recommendation and cost-saving measures. There is no definitive scientific evidence proving that reusing phaco tubing will harm patients.

Aim: To assess if phacoemulsification tubing remains sterile during sequential phacoemulsification.

Setting: The study was conducted at Edendale Hospital and Greys Hospital in PMB. Greys Hospital has a single-use policy and at Edendale policy, multi-use of tubings is allowed.

Methods: This was an observational descriptive prospective study observed over 4 months. Routine phacoemulsification was carried out at each hospital as per hospital guidelines. At the end of the surgery, the tips of the tubing were cut off, placed in a standard specimen container and sent to the laboratory for culture and microscopy. Results were compared and two unused tubings were also analysed as controls.

Results: A total of 26 single-use tubings were analysed and it was found that $46.2 \%$ grew no organisms; 19.2\% grew Bacillus species; other microorganisms were in less than $1 \%$ of tubings. A total of 41 multiple-use tubings were sent for analysis. Forty one and a half percent grew no organisms; $17.1 \%$ tubings grew Bacillus species. Other microorganisms were found in less than $5 \%$ of tubings. Unused tubings did not grow any microorganism.

Conclusion: A $p$ value less than $0.05 \%$ was accepted as statistically significant. Comparing the two hospitals, tubings which grew no organisms were $46.2 \%$ (single-use) versus $41.5 \%$ (multiple-use). This gives a statistically non-significant $p$ value of 0.70 . Tubings which grew Bacillus species were 19.2\% (single-use) versus $17.1 \%$ (multiple-use) $(p=0.83)$. Other microorganisms also showed a $p$ value of 0.76 . This implies that phacoemulsification tubing is significantly contaminated after just one use. Although no statistical difference was found between the two groups, a contamination rate of over $40 \%$ is of great concern. There are contributing factors which may have influenced this result. In our setting, phaco tubing does not seem to remain sterile after cataract surgery. Further research needs to be carried out in order to gain more insight into patient safety.

Keywords: cataract; phacoemulsification; microscopic analysis; single-use; multi-use.

\section{Introduction}

For patients, cataracts mean blurry vision, poor night vision, fading colours and haloes around lights. Phacoemulsification ('phaco') has become the leading technique of cataract extraction worldwide.

The first documented complete surgical extraction of the lens from the eye was in 1748 in Paris. ${ }^{1}$ Over the past few decades, major surgical improvements have been achieved by removing the cataract and leaving an intact posterior capsule behind. ${ }^{2}$ A decrease in the area of wound size and the introduction of sutures during surgical procedures greatly improved the success rate of cataract extraction. The Pietermaritzburg (PMB) complex comprises three hospitals. Two of them participated in the study. The third one did not offer phacoemulsification at the time of the study. All these hospitals do not have a uniform protocol regarding reuse of phacoemulsification tubing. Each hospital bases its protocol either solely on manufacture recommendation or a combination of manufacture recommendation and cost-saving measures. 
Manufacture guidelines state that one phacoemulsification pack must be used per patient (the tubing is part of the sterile 'phaco packs'). The Department of Health is looking for ways to save costs in the province, and proving that reuse of phacoemulsification tubing does not expose patients to more harm could be one way of contributing to cost-saving measures.

There is little scientific evidence evaluating whether reusing phaco tubing will harm patients. A previous study found that microorganisms could be transferred from infected to non-infected eyes during sequential phacoemulsification, but the study only analysed probes, irrigation and aspiration fluids.

The aim of this study is to assess if phacoemulsification tubing remains sterile during sequential phacoemulsification. The objectives are to investigate if there is growth of microorganisms from phaco tubing that are reused in sequential phacoemulsification at the two sites and to investigate and compare microorganisms, if identified, from each specimen (piece of sterile phaco tubing) that is sent for culture. In this study, probes are autoclaved before being reused, but the tubings are not. Microscopic analysis of the tubing should serve as evidence for drafting a standard protocol for the PMB complex and assess patient safety in general.

\section{Research method and design}

This study was an observational descriptive prospective study. It was carried out for over 4 months, from 31 March 2019 to 18 July 2019. It was conducted at Greys Hospital and Edendale Hospital. Routine phaco procedure was performed. Drapes, blades, knives and phaco probes were all sterilised before being reused. Reused tubings were not sterilised between cases. Slates and procedures were not altered for the study. We used these two hospitals because they currently have different protocols on used phacoemulsification tubings. At Greys Hospital, new phacoemulsification tubing was used for each case. At Edendale Hospital, phacoemulsification tubing was reused on three sequential patients on one slate. At the end of the case(s), just before the tubing was discarded (after each case at Greys Hospital and at the end of every third case at Edendale Hospital), a $5 \mathrm{~cm}$ piece of irrigation tubing tip was cut off, placed in a sterile specimen container and sent to the laboratory for investigation. Two unused irrigation tubings were also sent to the laboratory for testing as controls. At the laboratory, the specimens were incubated in a broth medium overnight and then were transferred on to Agar plates for culture.

\section{Study population and sampling strategy}

Theatre cases were booked as per standard departmental theatre slates. Slates are often booked months in advance. Specimens were collected as they were available at the end of the procedure(s).
Inclusion criteria: all irrigation tubing used during that period are to be kept sterile and reach the laboratory in a standard specimen container.

Exclusion criteria: all known contaminated or unsterile tubings, and tubings used in any other techniques of cataract extraction including lens washout, extracapsular lens extraction and intracapsular lens extraction are excluded as samples for this study.

The intended sample size was 76 phacoemulsification tubing from three hospitals in the PMB complex. This was an estimated number of phacoemulsification surgeries performed in the PMB complex in one month. One hospital did not offer the procedure at the time of the study due to lack of a surgeon so only two hospitals collected specimens, and the sample size was decreased to 67. Collection took longer than one month due to unforeseen circumstances. These included lack of phaco packs at Greys Hospital during that period and the theatre running out of colour coded specimen bottles at Edendale Hospital. Forty-one irrigation tubings from Edendale Hospital were analysed, and 26 tubings from Greys Hospital. Two unused tubings were analysed. The accuracy of our estimated average and range was calculated using statistical analysis.

\section{Data collection}

Standard theatre specimen bottles and laboratory forms were left in theatre. Surgery was performed by different surgeons. Before discarding the tubing, the surgeon cut the $5 \mathrm{~mm}$ piece with unused sterile scissors and inserted it into the specimen container. The specimen and the laboratory request forms were placed in clear laboratory plastic bags. Plastic bags, specimen bottles and request forms for the study were colour coded for easy identification of the hospital it originated from. All used phacoemulsification irrigation tubing was cultured and microscopically analysed.

\section{Data analysis}

The $\mathrm{Z}$ test, which tests for a difference of two proportions, was used for statistical analysis. The $p$ value of less than $0.05 \%$ was taken as statistically significant. We calculated the confidence level using the following formula:

Confidence level $=\mathrm{P} \pm 1.96(\mathrm{P})(1-\mathrm{P}) / \mathrm{n}$

[Eqn 1]

\section{Ethical considerations}

Ethical approval to conduct the study was obtained from Greys Hospital, Edendale Hospital, UKZN Biomedical Research Ethics Committee, KZN Health Research Committee (Ethical Clearance Number: 2048990).

Patients were not enrolled for the study. No patient consent or record was required. The state laboratory was used to analyse our phacoemulsification tubing. This will benefit patients in future as the recommendations are made to 
improve patient safety and/or improve cost-effectiveness. Conflict of interest may be present, as this study is conducted only towards a certain degree.

\section{Results}

Phacoemulsification irrigation tubing was sent for microscopic analysis to show whether they remain sterile during sequential phacoemulsification. The results, as shown in Table 1, show that not all tubing remains sterile after phacoemulsification. Less than half of the tubings stay sterile as no microorganisms were found in $41.5 \%$ (Edendale) and $46.2 \%$ (Greys). Tubings that did not remain sterile grew a variety of microorganisms as shown in Table 1. Most types of microorganisms grew on only one or two tubing in each hospital. Bacillus species grew in multiple (5 or more) tubings. In comparing the two hospitals, clinically, Greys Hospital had more tubings which showed no growth of microorganisms than Edendale Hospital, but the difference was only $4.7 \%$. Unused tubings did not grow any microorganisms.

The $\mathrm{Z}$ test, which compares the difference of two proportions, was used for statistical analysis. The $p$ value of less than $0.05 \%$ was taken as statistically significant. We calculated the confidence level using the formula:

Confidence level $=\mathrm{P} \pm 1.96(\mathrm{P})(1-\mathrm{P}) / \mathrm{n}$

[Eqn 2]

Comparing the two hospitals, tubing which grew no organisms were $46.2 \%$ (single-use) versus $41.5 \%$ (multipleuse). This showed a statistically non-significant $p$ value

TABLE 1: Organisms cultured per study site.

\begin{tabular}{|c|c|c|c|c|}
\hline \multirow[t]{2}{*}{ Organism } & \multicolumn{2}{|c|}{$\begin{array}{l}\text { Greys Hospital } \\
\text { (single-use) }\end{array}$} & \multicolumn{2}{|c|}{$\begin{array}{l}\text { Edendale Hospital } \\
\text { (multiple-use) }\end{array}$} \\
\hline & $n$ & $\%$ & $n$ & $\%$ \\
\hline Acinobacter baumanii complex & - & - & 2 & 4.9 \\
\hline Bacillus species & 5 & 19.2 & 7 & 17.1 \\
\hline $\begin{array}{l}\text { Coagulase-negative Staphylococcus } \\
\text { and Micrococcus }\end{array}$ & - & - & 1 & 2.4 \\
\hline Corynebacterium species & 1 & 3.8 & 1 & 2.4 \\
\hline Granulicatella adiacens & 1 & 3.8 & - & - \\
\hline Klebsiella pneumonia & - & - & 2 & 4.9 \\
\hline Micrococcus luteus & - & - & 2 & 4.9 \\
\hline Micrococcus species and Bacillus species & 1 & 3.8 & - & - \\
\hline Myroides species & - & - & 1 & 2.4 \\
\hline Pantoea species & - & - & 1 & 2.4 \\
\hline $\begin{array}{l}\text { Pseudomonas stutzeri and Coagulase } \\
\text { neg Staphylococcus }\end{array}$ & 1 & 3.8 & - & - \\
\hline Rhizobium radiobacter & 1 & 3.8 & - & - \\
\hline Serratia plymuthica & 1 & 3.8 & - & - \\
\hline Sphingomonas paucimobilis & - & - & 2 & 4.9 \\
\hline $\begin{array}{l}\text { Staphylococcus auerues and } \\
\text { Staphylococcus lentus }\end{array}$ & - & - & 1 & 2.4 \\
\hline Staphylococcus capitis & - & - & 1 & 2.4 \\
\hline Staphylococcus cohnii & 1 & 3.8 & - & - \\
\hline Staphylococcus epidermidis & 1 & 3.8 & - & - \\
\hline $\begin{array}{l}\text { Staphylococcus hominis and } \\
\text { Sphingomonas paucimobilis }\end{array}$ & - & - & 1 & 2.4 \\
\hline Staphylococcus warneri & - & - & 1 & 2.4 \\
\hline Staphylococcus salivarius & 1 & 3.8 & 1 & 2.4 \\
\hline No growth & 12 & 46.2 & 17 & 41.5 \\
\hline Total & 26 & 100 & 41 & 100 \\
\hline
\end{tabular}

of 0.70394. Tubing which grew Bacillus species were 19.2\% (single-use) versus $17.1 \%$ (multiple-use) $(p=0.83)$, confidence level of $17.1 \%$ - $19.2 \%$ (single-use), versus confidence level of $14.52 \%$ - 19.68\% (multiple-use). Tubings which grew Bacillus species displayed a $p$ value of 0.83 . On comparing a variety of other microorganisms, the $p$ value of 0.76 was obtained which is statistically also not significant. The 95\% confidence level showed us a true value of an unknown population.

\section{Discussion}

The introduction of phacoemulsification has shortened surgery time and improved wound healing, but the cost of each surgery has risen drastically. In South Africa, the cost of one cataract extraction in private practice is slightly below R30 000.00 (\$400.00). In the state hospitals, we have managed to reduce the cost of consumables to R2500.00 primarily due to special pricing and reuse of items.

Hospitals that participated in our study included Greys Hospital, a tertiary level hospital, where they use a single pack per patient and Edendale Hospital, a secondary level hospital, where they reuse phacoemulsification packs. A microscopic analysis of tubings from these hospitals showed that more than $50 \%$ of tubings grew bacteria after use but the virulence and number of organisms did not grow with multiple-use. Over $40 \%$ of tubings from both hospitals did not grow bacteria.

The discovery of safe and cost-effective surgical techniques is very important especially in the developing world. The practice of reusing single-use devices is carried out worldwide. In America, it is subject to Food and Drug Administration oversight. ${ }^{3}$ Although it is often considered to be safe and effective, little published evidence is available on safety and efficacy. ${ }^{4}$ In Australia, a study by infection control experts at the Woden Valley Hospital in Canberra indicated that reuse was occurring in $38 \%$ of all respondents. ${ }^{5}$ Reuse occurred in many large metropolitan hospitals (in 64\% of those with more than 300 beds) than in smaller metropolitan hospitals (in $41 \%$ of hospitals with fewer than 300 beds), or in private hospitals (32\%).

Post-phacoemulsification endophthalmitis is one of the most severe complications of cataract surgery, and it may lead to permanent blindness. Our study did not look into the rates and causes of post-phacoemulsification endophthalmitis. The growth of microorganisms can occur in almost any step during surgery. Studies have found that wound incisions are the leading pathway for introducing flora into the eye. Lack of equipment sterility is a wide area where microorganisms can be introduced. Equipment is therefore often prepacked to ensure quicker access and usage whilst maintaining sterility.

All state hospitals in the PMB complex utilise the Infinity ${ }^{\mathrm{TM}}$ system (Alcon Laboratories) which employs a single-use pack for each procedure. Each sterile pack consists of a cartridge with irrigation fluid attachments (which fits into 
the phaco machine), as well as tubing, which fits into the autoclaveable hand piece. Only the hand piece is in contact with the patient's eye. The cartridge and tubing could therefore theoretically remain sterile for many cases if handled with care. Based on this theory, our study looked into finding microscopic evidence of whether tubings remain sterile after use.

A study in the European Journal of Ophthalmology in 2012 aimed to determine if there was microbial contamination of the irrigating fluids at the time of phacoemulsification after the use of topical povidone-iodine and antibiotics prophylaxis. ${ }^{7}$ After each case, fluid was collected and sent for microbiology analysis. Results showed that there was indeed contamination of irrigating fluid, but preoperative use of antibiotics decreased the rate of endophthalmitis infection to practically zero. We found similar results in our study. More than half of the phaco tubings are contaminated after use, even it is used on a single patient only. This is due to a variety of factors. Contamination of the tubings could have occurred during handling, from the time the tubings were cut and placed inside the specimen bottles to the time they were prepared for microscopy in the laboratory. The specimen bottles themselves could have been the source of contamination, as they are not sterile bottles although they are the standard theatre specimen bottles. There are no previous studies that have looked specifically into reusing phacoemulsification irrigation tubing in isolation. A study was conducted in Brazil that looked into viral contamination during sequential phacoemulsification surgeries in an experimental model. The purpose of the study was to determine the incidence of Piry virus contamination among surgical instruments used with disposable accessories for phacoemulsification during sequential surgeries. ${ }^{8}$ Four pigs eyes were contaminated with the Piry virus and the other four eyes were not. Phacoemulsification surgeries were performed on all eight eyes alternating between contaminated and non-contaminated eyes. The hand piece, irrigation and aspiration tubes were reused; the operating fields, gloves, scalpels, tweezers, needles, syringes, tip and bag collector from the phacoemulsification machine were exchanged. In their study, they analysed specimens from the collector bags, the tips, irrigation, and aspiration system. From their irrigation system, a sample from a noncontaminated eye (one-fourth) was found positive and in their aspiration system two samples from non-contaminated eyes were found positive. In the collector bag, two samples from non-contaminated eyes were positive. At the tip, two samples from non-contaminated eyes were positive. They also found two samples from the anterior chamber of non-contaminated eyes to be positive post-surgery. The conclusion was that there was transfer of genetic material of the Piry virus during sequential phacoemulsification where the tip, irrigation and aspiration systems were reused between surgeries. ${ }^{9}$

In our study more than half of our tubings grew bacteria in both groups (see Table 1). We attributed this to contamination.
A variety of bacteria were cultured. Bacillus species was the most prevalent contaminant. All other species identified have never been associated with endophthalmitis. Bacillus species have been known as opportunist pathogens since the late 19th century. Separation of this pathogen cannot be performed in isolation without taking the clinical picture into account. ${ }^{10}$ Certain subtypes of Bacillus like Bacillus anthracis and Bacillus cereus are associated with terminal illnesses. B. cereus causes destructive intraocular damage, severe keratitis, conjunctivitis, iridocyclitis, panophthalmitis, dacrocystitis and orbital abscess. Our patients were all clinically asymptomatic before and after surgery, so the deadly and sight threatening subtypes of Bacillus are less likely to be the ones isolated. This does not, however, negate the fact that more than half of the phaco tubing was contaminated.

Staphylococcus epidermidis has previously been regarded as an innocuous commensal microorganism on the human skin, but nowadays it is seen as an important opportunistic pathogen. It is now the most frequent cause of nosocomial infections, at a rate about as high as that due to its more virulent cousin Staphylococcus aureus. ${ }^{11}$ Staphylococcus epidermidis is the most common source of infections on indwelling medical devices. This results from the fact that it is a permanent and ubiquitous coloniser of human skin, and the device becomes contaminated during use. ${ }^{12}$ Staphylococcus epidermidis infections only rarely develop into life-threatening diseases.

Corynebacterium diphtheria is linked to epidemic outbreaks in Russia in the 1990s. It has since then seen a decline. Today, the more common scenario is non-diphtherial Corynebacterium bacteremia associated with device infections (venous access catheters, heart valves, neurosurgical shunts, peritoneal catheters), as well as meningitis, septic arthritis and urinary tract infections. ${ }^{13}$

There have been cases of post-operative endophthalmitis associated with equipment contamination. A study was carried out in London whose purpose was to set up a model for the assessment, investigation and management of an atypical outbreak of infectious endophthalmitis of unknown cause in London in 2003. ${ }^{14}$ A multidisciplinary infection control team was formed with the aim of identifying potential causative factors. These factors included analysing the theatre and its surrounding environment, preoperative preparation, intraoperative theatre and surgical practices, post-operative practices, equipment maintenance guidelines, cleaning and sterilisation practices and microbiological screening. Five cases of endophthalmitis following uncomplicated phacoemulsification, by different surgeons, were noted over a 7-month period. Three cultures grew Streptococcus viridans of different strains, one culture grew $S$. aureus, and no organisms grew on the last culture. Without a single causative factor, it was postulated that it was a combined effect of many possible factors that led to increased bacterial load and subsequent infection rate. Recommendations were made which included new cleaning protocols to prevent the build-up of debris on the 
phacoemulsification tubing. In our study, more than half of our tubings were contaminated (Table 1) after surgery. This is clinically significant, although many were innocuous microorganisms. The results of single versus multiple-use devices were however not statistically significant. These clinical results warrant further research in this field.

\section{Strengths}

The actual culture and microscopic analysis were carried out at a single recognised laboratory by qualified laboratory staff under the supervision of a qualified pathologist.

The study was conducted in a real-world setting without changes to any of the procedures or methods, and therefore, gives an accurate reflection of what is currently happening in our theatres.

\section{Limitations}

Sample size was kept small primarily due to cost. A larger sample size could have had a more representative result. Duration was also a limiting factor, if more tubing were analysed over a long period a more representative result may have been obtained.

Different laboratory staff analysed the specimen. This could mean different technique and lack of proper insight of the study by the different laboratory technicians.

Different theatre teams may also contribute to study limitation due to possible different specimen handling and sampling.

All tubings were from the same company. A comparison of tubings from different companies may have showed a different result.

\section{Implications and recommendations}

This study has laid a foundation for future studies on reusing phacoemulsification tubing.

Phacoemulsification tubing seems to be contaminated even after only one use despite our efforts to keep the tips sterile during and between procedures. We recommend further research in this field with a larger sample size, one surgeon, use of a sterile and not a standard specimen container, one laboratory technician and a specialist pathologist. This study has not proven sterility of phaco tubings even with single use, so safety of patients cannot be guaranteed. Further research in this field is warranted.

\section{Conclusion}

Microscopic analysis has shown that there may be growth of microorganisms in tubings whether they have been used only once or multiple times. Unused tubings were confirmed sterile as no growth of microorganisms was found. No statistically significant difference between single-used and multi-used tubing was found. Less than half of tubings remained sterile after single and multi-use. Clinically, the high rate of contamination is of great concern. Based on this study, multiple reuse of phacoemulsification tubing does not put patients at a much higher risk of infection than postsingle-use. After both single-use and multiple reuse, safety was not guaranteed due to the high contamination rate. Tested preused tubings were not found to be contaminated. Further studies need to be performed to gain more insights into patient safety.

\section{Acknowledgements}

The author thanks Dr C. Kruse for his guidance, Dr R. Rodseth for his valuable input in preparing the protocol, and Dr B. Tlou for the statistical analysis in this study.

\section{Competing interests}

The author has declared that no competing interests exist.

\section{Authors' contributions}

L.T.N. is the sole author and is responsible for literature review, data collection and analysis.

\section{Funding information}

The study was funded by the author.

\section{Data availability statement}

Data sharing is not applicable to this article.

\section{Disclaimer}

The views and opinions expressed in this article are those of the author and do not necessarily reflect the official policy or position of any affiliated agency of the author.

\section{References}

1. Davis G. Cataract surgery in the modern era. Am Acad Ophthalmol [serial online] 2008 [cited 2008 May 25]. Available from: http://www.aaofoundation.org/what/ heritage/exhibits/online/cataract/modern.cfm.

2. Agapitos PJ. Cataract surgical techniques. Curr Opin Ophthalmol. 1991;2(1):16-27. https://doi.org/10.1097/00055735-199102000-00003

3. Hopkins $\mathrm{P}$, Patel $\mathrm{S}$. Beware the Trojan horse-a timely reality check about reusing single-use devices. Anaesthesia. 2017;72(1):3-16. https://doi.org/10.1111/anae. 13523

4. Emergency Care Research Institute. Use of reprocessed single-use medical devices. Operating room risk management. Vol. 2. Technology management 11. Plymouth Meeting: ECRI Institute, University of Nottingham; 2009.

5. Collignon PJ, Graham E, Dreimanis DE. Reuse in sterile sites of single-use medical devices: how common is this in Australia? Med J Aust. 1996;164(9): 533-536.

6. Harrigan P. Re-use of single-use device in Australia. Lancet. 1996;347(9013):1476 https://doi.org/10.1016/S0140-6736(96)91708-0

7. Balestrazzi A, Malandrini A, Montagnani F, et al. Phacoemulsificator and sterile drapes contamination during cataract surgery: A microbiological study. Eur J Ophthalmol. 2011;22(2):188-194. https://doi.org/10.5301/ejo. 2011.8434

8. Coelho R, Garcia TV, Paula JS, et al. Viral contamination during sequential phacoemulsification surgeries in an experimental model. Arq Bras Oftalmol. 2012;75(3):174-177. https://doi.org/10.1590/S0004-27492012000300005 
9. Coelho RP, Garcia TV, Paula JS, et al. Viral contamination during sequential phacoemulsification surgeries in an experimental model. Arq Bras Oftalmol. phacoemulsification surgeries in an experimental model. Arq Bras Of
2012;75(3):174-177. https://doi.org/10.1590/S0004-27492012000300005

10. Logan NA. Bacillus species of medical and veterinary importance. J Med Microbiol. 1988;25(3):157-165. https://doi.org/10.1099/00222615-25-3-157

11. CDC. National Nosocomial Infections Surveillance (NNIS) System Report data summary from January 1992 through June 2004, issued October 2004. Am J Infect Control. 2004;32(8):470-485. https://doi.org/10.1016/j.ajic.2004. 10.001
12. Uckay I, Pittet D, Vaudaux P, Sax H, Lew D, Waldvogel F. Foreign body infections due to Staphylococcal epidermidis. Ann Med. 2009;41(2):109-119. https://doi. org/10.1080/07853890802337045

13. Frassetto LA. Cortynebacterium infections. [homepage on the Internet]. [cited 30 July 2018]. Department of Internal Medicine, San Fransisco: University of California, 2018, School of Medicine, Medscape.

14. Anderson OA, Lee $\mathrm{V}$, Shafi $\mathrm{S}$, et al. A model for the management of an atypical endophthalmitis outbreak. Eye. 2004;19(9):972-980. https://doi.org/10.1038/ sj.eye.6701695 